\title{
Low temperature compressive creep in electrodeposited nanocrystalline nickel
}

\author{
Ravi S. Kottada, Atul H. Chokshi * \\ Department of Metallurgy, Indian Institute of Science, Bangalore 560 012, India
}

\begin{abstract}
Steady state creep was not observed during large strain compressive creep in electrodeposited nanocrystalline-Ni. An additional exothermic peak during differential scanning calorimetry of deformed samples is attributed to recovery associated with geometrically necessary dislocations. The lack of change in texture suggests that grain boundary sliding and rotation occurred during creep.
\end{abstract}

Keywords: Nanocrystalline; Nickel; Creep; Dislocation; Diffusion

\section{Introduction}

Since grain boundaries limit intragranular dislocation motion, it is well known that a reduction in grain size to the nanocrystalline regime offers a potent means of strengthening materials [1].

However, there are experimental data as well as computational simulations that suggest that at very fine grain sizes, a reduction in the grain size will lead to a reduction in strength due either to diffusion creep processes, some form of grain boundary sliding, or limitations in the operation of dislocations [2-4].

Creep deformation is usually characterized by the following expression [5]:

$\dot{\varepsilon} \propto D d^{-p} \sigma^{n}$

where $\dot{\varepsilon}$ is the steady state strain rate, $D$ is the appropriate diffusion coefficient, $d$ is the grain size, $\sigma$ is the imposed stress, and $p$ and $n$ are constants termed the inverse grain size and stress exponent, respectively. The diffusion coefficient is given as $D=D_{0} \exp (-Q / R T)$,

\footnotetext{
* Corresponding author.

E-mail address: achokshi@met.iisc.ernet.in (A.H. Chokshi).
}

where $D_{0}$ is a pre-exponential term, $Q$ is the appropriate activation energy, and $R$ is the gas constant. Typically, intragranular dislocation creep processes involve $p=0$ and $n>3$, whereas grain boundary sliding and diffusion creep processes involve $n \leqslant 3$ and $p \geqslant 1$.

There have been two independent studies on creep in electrodeposited nanocrystalline Ni. Wang et al. [6] conducted experiments at room temperature and they reported $n \sim 1$ for a grain size of $6 \mathrm{~nm}$, and a transition from $n \sim 2$ to $n \sim 5$ for Ni with $d=20$ and $40 \mathrm{~nm}$. Yin et al. [7] reported $n \sim 1$ and $\sim 6$ for Ni with $d=30 \mathrm{~nm}$ tested at room temperature and at $373 \mathrm{~K}$, respectively. The experimental results with $n \sim 1$ were attributed to Coble grain boundary diffusion creep [8], whereas $n>5$ has been attributed to some form of dislocation creep. The activation energy for creep was not measured.

Examination indicates that the total strains during early creep studies were very small (typically $<0.5 \%$ ), and they may not relate to steady state deformation. Since models for creep are based on steady state deformation, there is a need to re-examine experimental results on nanocrystalline materials. The relatively low tensile ductility of nano-Ni at low temperature is a serious limitation. While rolling experiments can provide 
large strains, the flow stresses are usually not very well known $[9,10]$. Uniaxial compression offers a simple means for overcoming the above limitations.

\section{Experimental material and procedures}

Electrodeposited $\mathrm{Ni}$ plates with a thickness of $1.75 \mathrm{~mm}$ and square dimensions of $9 \mathrm{~mm} \times 9 \mathrm{~mm}$ were obtained using procedures described earlier [11]. Compression creep samples with a nominal square crosssection of $1.75 \times 1.75 \mathrm{~mm}$ and a height of $2.7 \mathrm{~mm}$ were cut from the as-received plates. The grain sizes and textures in the specimens were characterized using $\mathrm{X}$-ray diffraction (XRD) using $\mathrm{Cu} \mathrm{K} \alpha$ radiation. The grain sizes were obtained from the broadening of the (111) peak using the Scherrer formula, after subtraction for instrument broadening. The variation in grain size and texture was characterized by polishing the sample and conducting XRD sequentially. The grain sizes were also determined from a creep deformed sample, as well as a sample annealed for the same time as the creep test.

Constant load compression creep tests were conducted at $\sigma=1000-2000 \mathrm{MPa}$ and $T=300-400 \mathrm{~K}$; most of the experiments were carried out at $T=373 \mathrm{~K}$. The specimens were loaded to the required initial stress within $2 \mathrm{~min}$, after the stabilization of the experimental setup for $1 \mathrm{~h}$ at the testing temperature. Deformation was quite uniform, without significant barreling. A few experiments were conducted at $T=353$ and $393 \mathrm{~K}$ to evaluate the activation energy for deformation. A temperature cycling experiment was also conducted for determining the activation energy; the experimental setup required the removal of load during the interval necessary for stabilizing the system at the altered temperature.

Differential scanning calorimetry (DSC) experiments were carried out on the as-received sample and on a few deformed samples, using a heating rate of $10 \mathrm{~K} \mathrm{~min}^{-1}$.

\section{Experimental results}

\subsection{Microstructural considerations}

The variation in texture (defined here as the relative ratio of $I_{(111)} / I_{(200)}$ peaks) and grain size through the thickness of the electrodeposited plate, shown in Fig. 1a, depicts a progressive increase in grain size and texture across the thickness of the plate. Fig. 1b illustrates the influence on grain growth and texture of annealing and creep deformation at $\sigma=2 \mathrm{GPa}$ and $\varepsilon \sim 28 \%$; the open and filled bars correspond to the two opposite sides of the electrodeposited plate.

The data suggest that grain growth is enhanced by deformation. Annealing and creep at $373 \mathrm{~K}$ did not
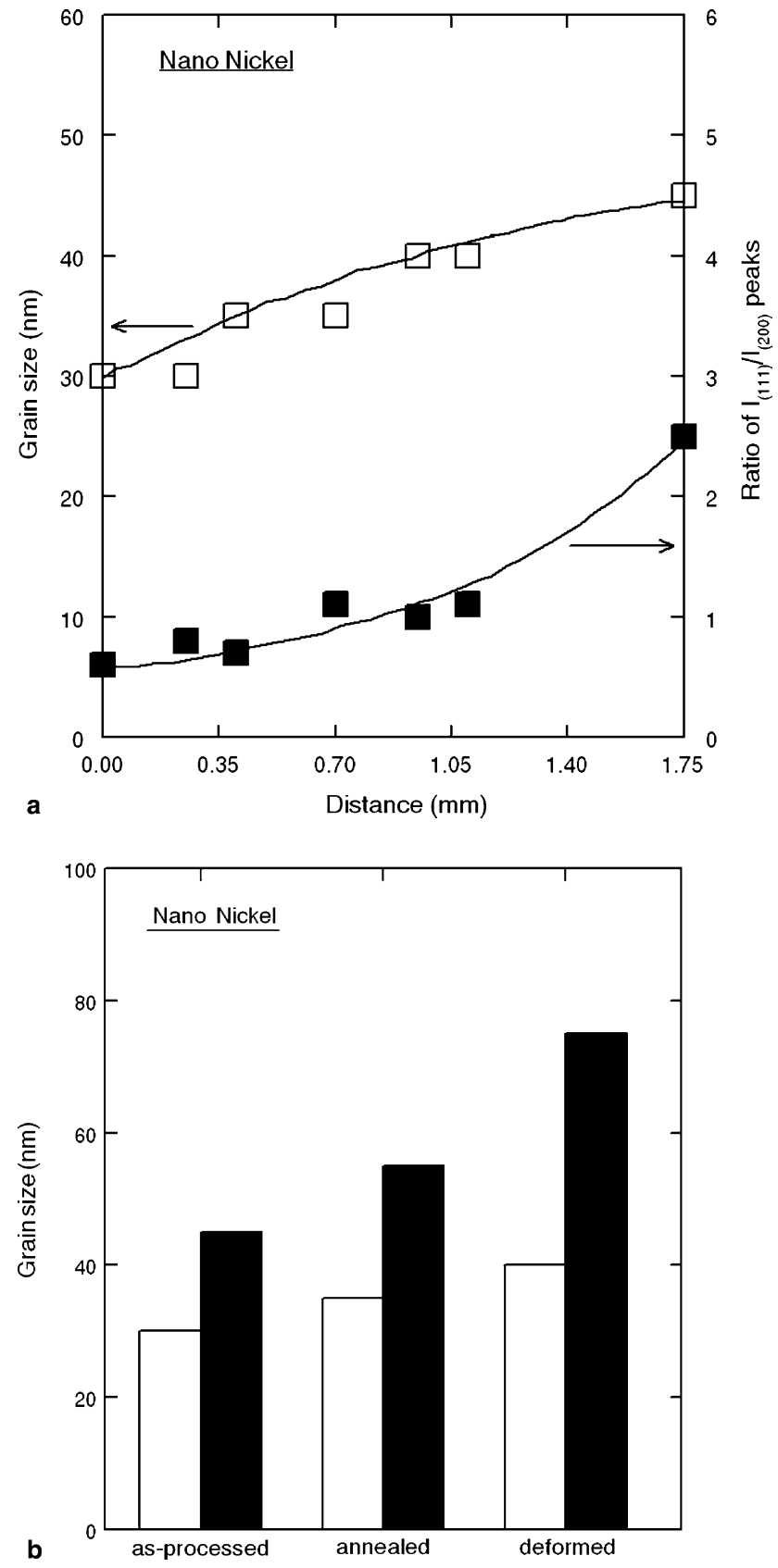

Fig. 1. (a) Variation of grain size and texture across the thickness of electrodeposited plate. (b) Comparison of grain size in the as processed, annealed $(44 \mathrm{~h}$ at $373 \mathrm{~K}$ ) and deformed (at $373 \mathrm{~K}$, at 2 GPa for $28 \%$, for $\sim 42 \mathrm{~h}$ ) specimens.

affect the texture, with the ratios of $I_{(111)} / I_{(200)}$ peaks retaining the initial values of $\sim 0.6$ and 2.5 at two surfaces of the samples.

\subsection{Thermal analysis}

Fig. 2 shows the DSC curves for the as-received sample and a deformed sample. The as-received sample showed an exothermic peak at a temperature of $\sim 600 \mathrm{~K}$ and an endothermic peak at $620 \mathrm{~K}$; re-heating 


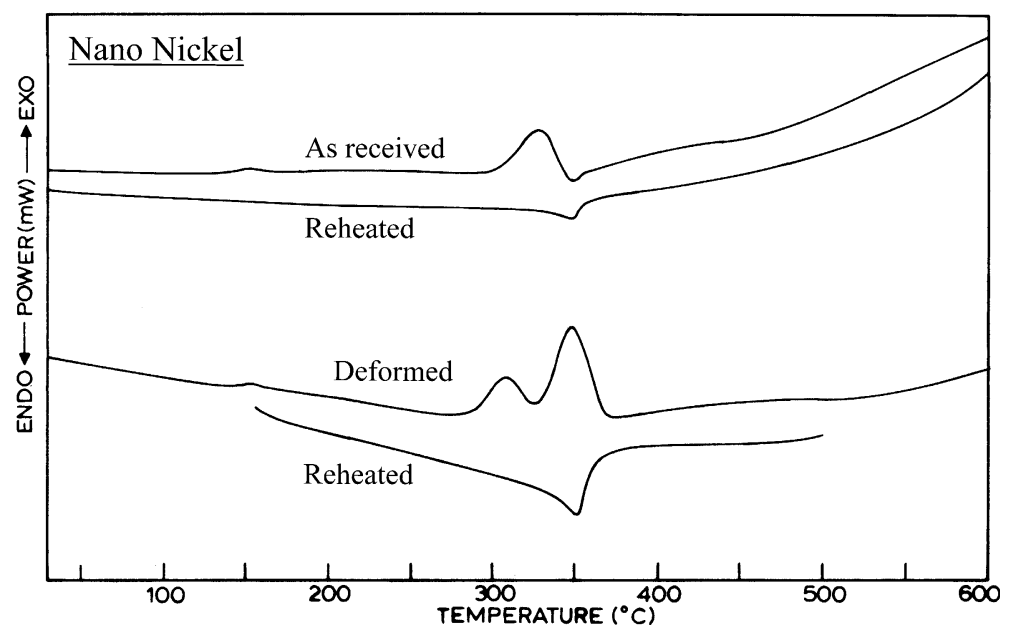

Fig. 2. DSC curves of the as received (top two curves) and deformed (two curves at the bottom) samples showing the extra exothermal peak in the deformed specimen at $\sim 580 \mathrm{~K}$.

the sample after cooling to room temperature led to a single endothermic peak at $620 \mathrm{~K}$. The exothermic peak observed in the as-received condition is usually identified with grain growth in nanocrystalline $\mathrm{Ni}$, and the endothermic peak has been related to a magnetic transition. It is interesting to note that there are two exothermic peaks at $580 \mathrm{~K}$ and $620 \mathrm{~K}$ after creep deformation. Two exothermic peaks were obtained also on two other specimens deformed at $373 \mathrm{~K}$ and a sample tested at room temperature; the first peak involved an energy release of $\sim 0.6$ to $1 \mathrm{Jg}^{-1}$. A double peak, related to abnormal grain growth [11], for (111) and (200) reflections was observed during XRD of a sample after the exothermic peak in the as-received condition. The lack of a double peak after the first DSC exothermic peak in the deformed specimen suggests that this peak is not related to significant abnormal grain growth.

\subsection{Mechanical data}

Fig. 3a shows the variation in creep strain with time, and Fig. $3 b$ shows the corresponding variation in creep rate with strain. Although a cursory examination of Fig. 3a may suggest that steady-state creep was attained, inspection of Fig. $3 \mathrm{~b}$ indicates that the creep rates decrease even at large strains of up to $28 \%$. A similar analysis suggests that previously reported data $[6,7]$ also correspond to primary creep.

The variation in strain rate with stress, shown in Fig. 4a using data at fixed strains of 1\%, 2\%, 3\% and $4 \%$, yields stress exponents of $n \sim 8,10,12$ and 12 , respectively. Also shown in Fig. 4 a is the theoretical prediction of the Coble creep mechanism (dashed line), which is discussed later. Fig. $4 \mathrm{~b}$ depicts the variation in strain rate with strain using a temperature cycling experiment, which leads to an activation energy of $\sim 115 \mathrm{~kJ} \mathrm{~mol}^{-1}$. Analysis of data from independent tests at different temperatures also yielded activation energies of $\sim 90 \mathrm{~kJ} \mathrm{~mol}^{-1}$, at a constant strain of $3 \%$.

\section{Discussion}

The decrease in grain size with reduced texture observed in the present investigation is consistent with a previous report by Morgan et al. [12]. The experimental data in Fig. 1b provide evidence for deformation enhanced concurrent grain growth, in agreement with a recent molecular dynamic simulation [13].

\subsection{Strain hardening and accumulation of strain energy}

In creep involving grain boundaries, the inverse grain size exponent varies from 1 to 3 . However, the limited grain growth cannot account for the substantial decrease in the creep rate by over two orders of magnitude.

In constant load compression, the creep rate will decrease with strain $\dot{\varepsilon} \propto \exp \varepsilon^{n}$; for steady state, the slopes of the strain rate-strain plots should be constant. The changes in slopes of the curves in Fig. $3 \mathrm{~b}$ imply that there is no steady state for strains $<20 \%$. Nevertheless, calculations suggest that $n<20$ at high strains of $>20 \%$, which is consistent with results shown in Fig. 4a.

Recent calculations by Asaro and Suresh [14] have shown that for $\mathrm{Ni}$ with grain sizes $>15 \mathrm{~nm}$, deformation will occur by the movement of complete intragranular dislocations.

In face-centred cubic pure metals deforming by intragranular dislocations, a significant primary creep region is usually associated with the formation of a subgrain structure $[5] ; \lambda / b=20(G / \sigma)$ where $\lambda$ is the subgrain size, $b$ is the magnitude of the Burgers vector and $G$ is the shear modulus. Putting typical values of $\mathrm{G} \sim 79 \mathrm{GPa}$, $b=0.25 \mathrm{~nm}$ and $\sigma=2 \mathrm{GPa}$, calculations yield a value 

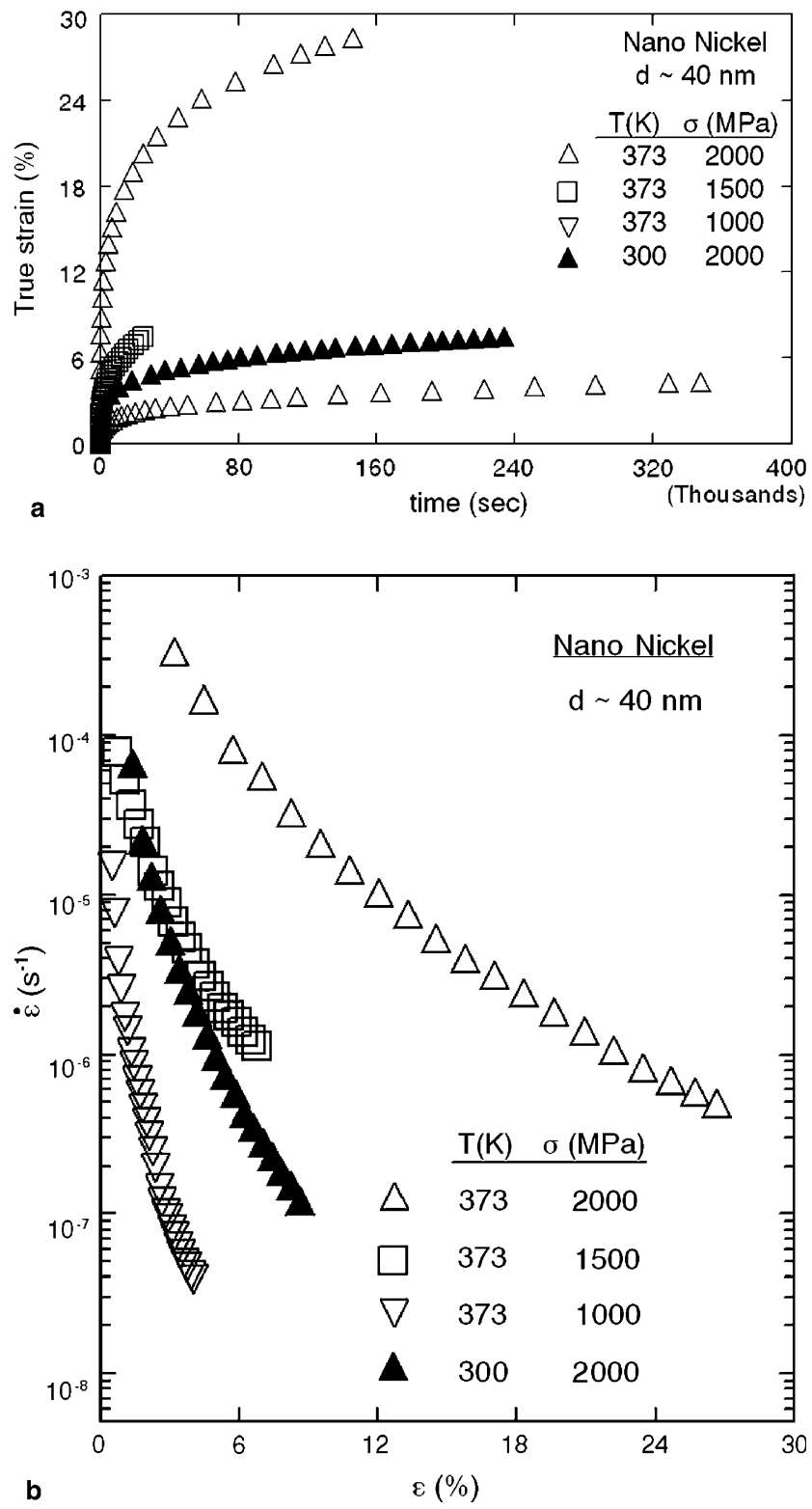

Fig. 3. (a) Creep curves at 300 and 373 K. (b) Strain rate vs strain curves for 300 and $373 \mathrm{~K}$.

of $\lambda \sim 200 \mathrm{~nm}$, so that the extensive primary creep cannot be attributed to subgrain formation.

Development of an intragranular dislocation network or mechanical twinning can also lead to strain hardening. However, experimental observations on deformed specimens do not show the development of any intragranular dislocation network or significant twinning in electrodeposited $\mathrm{Ni}$ with a grain size of $\sim 40 \mathrm{~nm}$ [3].

It may be possible to account for both strain hardening as well as energy storage during creep using the concept of geometrically necessary dislocations, $\rho_{\mathrm{g}}$, related to accommodating strain incompatibilities at grain boundaries [1]:

$\rho_{\mathrm{g}}=\bar{\varepsilon} / 4 b d$
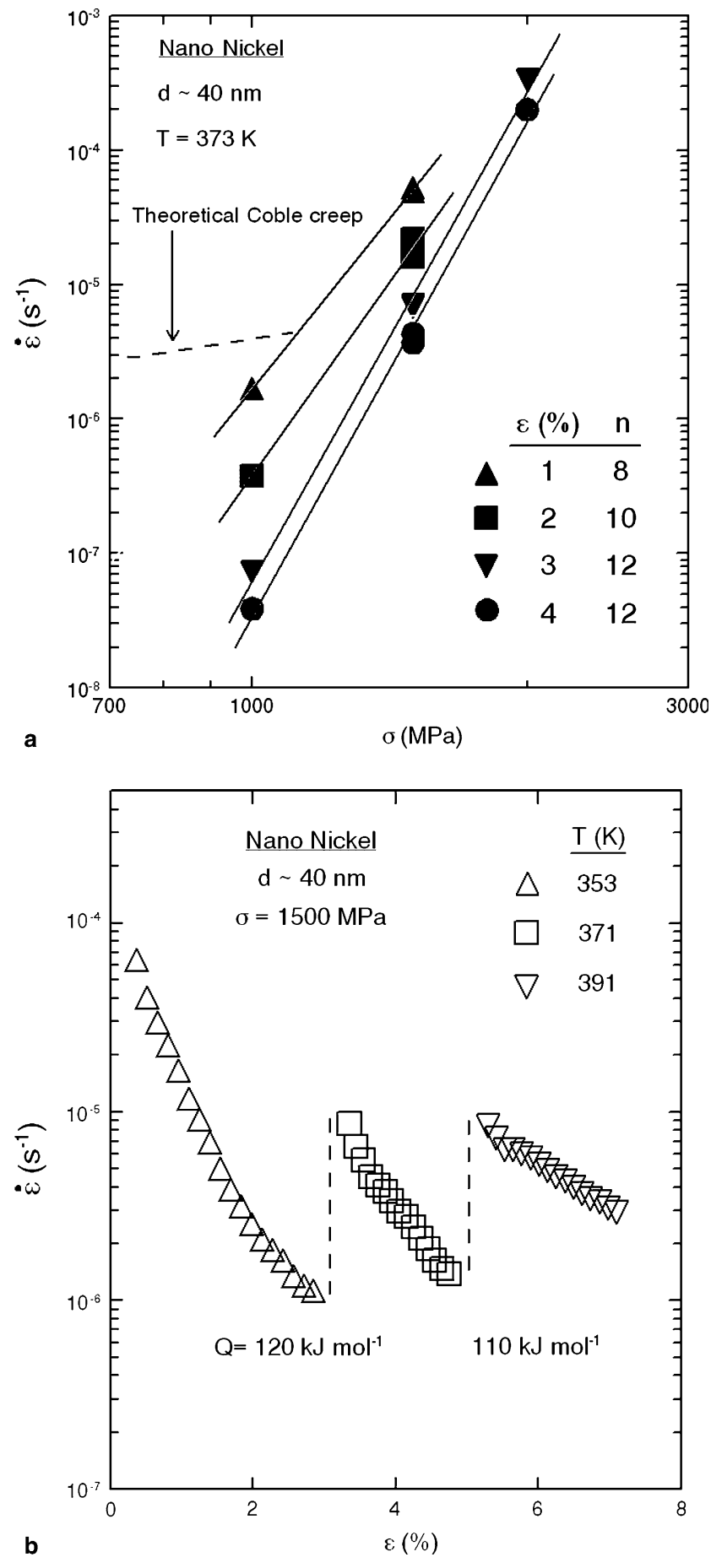

Fig. 4. (a) Variation of strain rate with stress. (b) Data from a temperature jump experiment.

where $\bar{\varepsilon}$ is the average strain. Calculation for a strain of 0.04 and a grain size of $40 \mathrm{~nm}$ yields a value of $\rho_{\mathrm{g}} \sim 10^{15} \mathrm{~m}^{-2}$. A similar approach was utilized earlier for deformation in coarse grained $\mathrm{Ni}$ [15].

During the first exothermic peak at $580 \mathrm{~K}$ after creep, the DSC measurements yielded an energy release of $\sim 0.6$ to $1.0 \mathrm{Jg}^{-1}$, Fig. 2. The release of strain energy 
stored during deformation $E_{\text {strn }}$ due to dislocations is [1]:

$$
E_{\text {strn }}=\left[G b^{2} \rho\right] /[4 \pi(1-v)]
$$

Using appropriate values for $\mathrm{Ni}$ in the above equation and $E_{\text {strn }} \sim 0.6$ to $1 \mathrm{Jg}^{-1}$, the dislocation density $\rho$ was estimated to be $\sim 10^{15} \mathrm{~m}^{-2}$. It is interesting to note that this value is of a similar order of magnitude as the geometrically necessary dislocation density calculated from Eq. (2).

Based on the above discussion, there are two possible explanations for the observed strain hardening. The development of a geometrically necessary dislocation content can lead to grain boundary hardening as envisioned, for example, by $\mathrm{Fu}$ et al. [16]. This hardening can lead to a reduction in the ability of grain boundaries to emit intragranular dislocation, which is reflected as strain hardening. Alternatively, it is possible that favorably oriented grain boundaries get exhausted during plastic deformation, so that strain hardening is caused by the need to operate stronger sources.

Budrovic et al. [17] noted an increase in peak broadening during tensile loading and a decrease in the initial value after unloading, suggesting that there was no accumulation of dislocations during deformation. While they reported very little strain hardening, the present compression experimental results indicate substantial strain hardening. It is possible that the reported lack of strain hardening is related to limited tensile ductility in nanocrystalline Ni.

\subsection{Rate controlling deformation processes}

Since Coble diffusion creep has been reported previously in some studies, the present experimental data were compared with the Coble creep rate $\dot{\varepsilon}_{\mathrm{Co}}$ [8]

$\dot{\varepsilon}_{\mathrm{Co}}=\frac{33 \delta D_{\mathrm{gb}}}{k T}\left(\frac{b}{d}\right)^{3} \sigma$

where $\delta$ is the grain boundary width and $D_{\mathrm{gb}}$ is the grain boundary diffusion coefficient, and $\delta D_{\mathrm{gb}}=4 \times 10^{-15}$ $\exp (-108,000 / R T) \mathrm{m}^{3} \mathrm{~s}^{-1}$ [18]. The dashed line in Fig. 3a indicates that Coble creep should be dominant at stresses below $\sim 1.5 \mathrm{GPa}$.

However, the experimental data do not follow the predictions of Coble creep, since the stress exponent $n$ is substantially greater than 1. Typically, Coble creep becomes interface controlled at critical stresses of $\sim G b / d$, which is $\sim 500 \mathrm{MPa}$ for Ni with $d=40 \mathrm{~nm}$.

The activation energies determined in the present investigation, $\sim 90-120 \mathrm{~kJ} \mathrm{~mol}^{-1}$, are close to the value for grain boundary diffusion. Finally, the lack of any change in texture after significant strains of $\sim 28 \%$, and grain growth, suggests that grain boundary sliding/rotation/migration must accompany intragranular dislocation creep, as reported also in nanocrystalline Pd [10].
Some models have been developed for grain boundary sliding and rotation deformation in nanometals $[19,20]$, related to superplasticity. The influence of $S$ on superplasticity, creep, grain boundary diffusion and grain growth is an area that merits additional attention [21-23].

\section{Conclusions}

Constant load compressive creep experiments on electrodeposited $\mathrm{Ni}$ with a grain size of $\sim 40 \mathrm{~nm}$ revealed extensive primary regions, and no evidence for steady state deformation. Differential scanning calorimetry measurements displayed an additional exothermic peak in deformed specimens, which is related to geometrically necessary dislocations. The lack of any change in texture coupled with grain growth suggests that grain boundary sliding/rotation/migration accompanies intragranular dislocation creep.

\section{Acknowledgement}

This work was supported by DST. We are grateful to Professor A. Cerezo of Oxford University for providing the material used in this study.

\section{References}

[1] Courtney TH. Mechanical behavior of materials. Singapore: McGraw-Hill; 1990.

[2] Mohamed FA, Li Y. Mater Sci Eng A 2001;298:1.

[3] Kumar KS, Van Swygenhoven H, Suresh S. Acta Mater 2003;51:5743

[4] Chokshi AH, Kottada RS. Trans Indian Inst Met, in press.

[5] Mukherjee AK, Bird JE, Dorn JE. Trans ASM 1969;62:155.

[6] Wang N, Wang Z, Aust KT, Erb U. Mater Sci Eng A 1997;237:150

[7] Yin WM, Whang SH, Mirshams R, Xiao CH. Mater Sci Eng A 2001;301:18.

[8] Coble RL. J Appl Phys 1963;34:1679.

[9] Lu L, Sui ML, Lu K. Science 2000;287:1463.

[10] Markman J, Bunzel P, Rosner H, Liu KW, Padmanabhan KA, Birringer R, et al. Scripta Mater 2003;49:637.

[11] Abraham M, Holdway P, Cerezo A, Smith GDW. Mater Sci Forum 2002;386-388:397.

[12] Morgan KL, Ahmed Z, Ebrahimi F. Mater Res Soc Symp Proc 2001;634:B3111.

[13] Haslam AJ, Moldovan D, Yamakov V, Wolf D, Phillpot SR, Gleiter H. Acta Mater 2003;51:2097.

[14] Asaro RJ, Suresh S. Acta Mater 2005;53:3369.

[15] Baker I, Liu L, Mandal D. Scripta Metall Mater 1995;32:167.

[16] Fu HH, Benson DJ, Meyers MA. Acta Mater 2004;52:4413.

[17] Budrovic Z, Swygenhoven HV, Derlet PM, Petegem SV, Schmitt B. Science 2004;304:273.

[18] Kaur I, Gust W, Kozama L. Handbook of grain and interphase boundary diffusion data. Stuttgart: Ziegler Press; 1989. p. 1014.

[19] Hahn H, Padmanabhan KA. Philos Mag B 1997;76:559. 
[20] Gutkin MY, Ovidko IA, Skiba NV. Acta Mater 2003;51:4059.

[21] Wang YM, Cheng S, Wei QM, Ma E, Nieh TG, Hamza A. Scripta Mater 2004;51:1023.
[22] McFadden SX, Mukherjee AK. Mater Sci Eng 2005;395:265.

[23] Dalla Torre F, Van Swygenhoven H, Schaublin R, Spatig P, Victoria M. Scripta Mater 2005;53:23. 